\title{
Tyrosine Hydroxylase in Vasopressinergic Axons of the Pituitary Posterior Lobe of Rats Under Salt-Loading as a Manifestation of Neurochemical Plasticity
}

\author{
Marina Abramova, ${ }^{1,2}$ André Calas, ${ }^{2}$ Jean Thibault ${ }^{3}$ and Michael Ugrumov ${ }^{1,2 \dagger}$ \\ ${ }^{1}$ Laboratory of Hormonal Regulations, Institute of Developmental Biology, Russian Academy of Sciences, \\ 26 Vavilov str., Moscow 117808, Russia; ${ }^{2}$ Département de Cytologie, Institut des Neurosciences, CNRS \\ UMR 7624, Université P. et M. Curie, 7 quai St. Bernard, F-75252 Paris Cedex 05, France; ${ }^{3}$ Laboratoire de \\ Neurobiologie moléculaire, Faculté des Sciences et Technologie, Université Paris 12-Val de Marne, \\ 61, avenue de Général de Gaulle, 94010 Créteil, France; ${ }^{8}$ Laboratory of Neurohistology, Institute of \\ Normal Physiology, Russian Academy of Medical Sciences, 8 Baltiiskaya str, Moscow, Russia
}

\section{SUMMARY}

In this study, we attempted to test whether tyrosine hydroxylase (TH), the first ratelimiting enzyme of catecholamine synthesis, is confined to the perikarya of activated magnocellular vasopressinergic (VPergic) neurons or is also present in their distal axons in the pituitary posterior lobe (PL). In addition, we evaluated the possible correlation between $\mathbf{T H}$ and VP turnover in the axons of rats drinking $2 \% \mathrm{NaCl}$ for 1,2 , and 3 weeks. To this aim, we examined the large swellings of VPergic axons, the so-called Herring bodies, using the doubleimmunofluorescent technique and the avidinbiotin technique, combined with image analysis. Here we have demonstrated for the first time a colocalization of TH and VP in Herring bodies, which is a strong argument in favor of $\mathrm{TH}$ transport from the perikarya of VPergic neurons via axons toward their terminals. THimmunoreactive (IR) and VP-IR materials were

Corresponding author:

Tel: +7095 1358842

Fax: +7 0951358012

e-mail: michael-ugrumov@mail.ru distributed in Herring bodies with seeming zonality. The number of VP-IR Herring bodies decreased by a factor of four over the first week of osmotic stimulation, remaining at almost the same low level until the end of the experiment. Conversely, the content of the VP-IR material within the individual Herring bodies fell gradually during the three weeks of saltloading. The results suggest that VP depletion from Herring bodies prevails in its transport into these structures during the whole period of osmotic stimulation. In contrast to VP-IR Herring bodies, the number of TH-IR Herring bodies and the content of TH-IR material within the individual Herring bodies increased progressively during the entire experiment. The synchronization of the VP depletion and TH accumulation in Herring bodies during longterm osmotic stimulation raised the question about a possible functional interaction between both substances.

\section{KEY WORDS}

Herring bodies, hypothalamo-postypophyseal system, salt-loading 


\section{INTRODUCTION}

Over the past two decades, many investigators (Brownstein \& Mezey, 1986; Hökfelt et al., 1987; Verney et al., 1988; Seil et al., 1992; Nagatsu et al., 1997) have demonstrated that some non-catecholaminergic neurons, mainly peptidergic neurons, express tyrosine hydroxylase (TH), the first ratelimiting enzyme of catecholamine synthesis. Vasopressinergic (VPergic) neurons of the supra-optic nucleus, projecting their axons to the posterior lobe (PL) of the pituitary, are considered one of the most promising cell models for studying this phenomenon. TH is expressed in VPergic neurons under functional stimulation (Kiss \& Mezey, 1986; Young et al., 1987; Meister et al., 1990a,b; Marsais \& Calas, 1999) following the perturbations of neural afferents (Kiss \& Mezey, 1986), during aging (Marsais et al., unpublished), and in pathology (Fetissov et al., 1997).

Taking into account that TH is a cytosolic nonsecreted protein, one might expect its accumulation in the perikarya of VPergic neurons during long-term osmotic stimulation, followed by its possible transport toward the axon terminals. However, a previous attempt to detect differences in TH-immunoreactivity (TH-IR) in the PL of intact and salt-loaded animals was unsuccessful (Meister, 1990b). Certain difficulties in detecting TH in VPergic axons should be related to a minor TH expression in VPergic neurons in intact animals and, conversely, to the rapid depletion of the axons from VP under salt-loading. Moreover, differentiating $\mathrm{TH}$-containing catecholaminergic and VPergic axons with a low VP levelundetectable with immunocytochemistry -is quite difficult or even impossible. Indeed, the PL is innervated by dopaminergic axons (Saavedra et al., 1975, Saavedra, 1985) belonging to the neurons of the periventricular (Kawano \& Daikoku, 1987) nucleus, as well as by noradrenergic fibers issued from the superior cervical ganglia (Saavedra, 1985; Vanhatalo et al., 1995).
The difficulties in detecting both substances in VPergic axons most probably could be overcome by studying Herring bodies, the large axon swellings that are generally considered a specific attribute of VPergic axons, but not of catecholaminergic axons (Dellmann \& Rodriguez, 1970; Polenov \& Garlov, 1971). In contrast to the relatively small swellings of the VPergic axons, Herring bodies partly retain VP under osmotic stimulation (Ugrumov \& Belenkii, 1974), namely, after the onset of TH synthesis in VPergic neurons (Kiss \& Mezey, 1986).

The present study attempted to test whether $\mathrm{TH}$ is an attribute not only of the cell bodies but also of the axons of magnocellular VPergic neurons, subsequently evaluating a possible correlation between TH and VP dynamic in Herring bodies during long-term salt-loading of the rats.

\section{EXPERIMENTAL PROCEDURES}

\section{Animals and Processing of Materials}

Adult male Wistar rats, weighing 200 to $300 \mathrm{~g}$ each, were used in this study. The animals were maintained at $21^{\circ} \mathrm{C}$ to $23^{\circ} \mathrm{C}$ in a light-dark cycle (light period between 8:00 and 20:00). Control (intact) rats had free access to food and tap water (nine animals), whereas the experimental rats were given $2 \% \mathrm{NaCl}$ instead of tap water for either 1 week (nine animals), 2 weeks (three animals), or 3 weeks (three animals). The rats were anesthetized with Pentobarbital $(50 \mathrm{mg} / \mathrm{kg}$ body wt) and perfused via the heart-first with saline for $10 \mathrm{~min}$ at $37^{\circ} \mathrm{C}$, and then with $4 \%$ paraformaldehyde in $0.1 \mathrm{M}$ sodium phosphate buffer $(\mathrm{pH} 7.3)$ for 15 $\min$ at $4^{\circ} \mathrm{C}$. Thereafter, the pituitaries were postfixed by immersion in the same fixative for $2 \mathrm{~h}$ at $4^{\circ} \mathrm{C}$, rinsed for $1 \mathrm{~h}$ in $0.02 \mathrm{M}$ phosphate buffer with $0.9 \% \mathrm{NaCl}$ (PBS, $\mathrm{pH} 7.2$ to 7.4 ) at $4^{\circ} \mathrm{C}$, immersed overnight in $15 \%$ sucrose PBS at $4^{\circ} \mathrm{C}$, 
and then frozen in isopentane at $-45^{\circ} \mathrm{C}$.

\section{Immunohistochemistry and Quantification}

In the first series of immunocytochemical studies, the pituitaries of intact rats and of rats that wer salt-loaded for $7 \mathrm{~d}$ ( 3 rats in each group) were used for TH and VP double-immunolabeling on the same cryostat sections, $14 \mu \mathrm{m}$ or $30 \mu \mathrm{m}$ thick. The sections were incubated overnight at $4^{\circ} \mathrm{C}$ in PBS containing a mixture of either (a) mouse monoclonal antibodies to TH (diluted 1:1000) (Sigma, USA) and rabbit polyclonal antibodies to VP (diluted 1:4000) (Chemicon, USA) or (b) mouse monoclonal anti-bodies to VP (diluted 1:20000) (Robert et al., 1985) and rabbit polyclonal antibodies to TH (diluted 1:2000), 1\% normal goat serum, and $0.1 \%$ Triton X-100. The sections were then incubated for $2 \mathrm{~h}$ at room temperature with a mixture of the secondary antibodies, rhodamine-conjugated goat anti-rabbit (diluted 1:40) (DAKO, Denmark) and fluoresceinconjugated goat anti-mouse (diluted 1:100) (Pasteur, France). The sections were rinsed twice in PBS, embedded in a mounting medium for fluorescence (Vectorlab, USA), coverslipped, and examined using either a fluorescence microscope (Leitz Diaplan, Germany) or a confocal microscope (Leica TCS 4D, Germany). The fluorescence microscope was equipped with filters for fluorescein-induced fluorescence $(450-490 \mathrm{~nm}$ excitation filter, $520-560 \mathrm{~nm}$ barrier filter, and 520-550 nm extra barrier filter) and for rhodamine-induced fluorescence $(546 / 10 \mathrm{~nm}$ excitation filter and $610 \mathrm{~nm}$ barrier filter). The confocal microscope was equipped with an argon/krypton laser $25 \mathrm{~mW}$ adjusted at $488 \mathrm{~nm}$ and $568 \mathrm{~nm}$ wavelengths for the respective excitations, as well as with filter sets for the selective detection of fluorescein $(488 \mathrm{~nm}$, green) and rhodamine $(568 \mathrm{~nm}$, red) fluorescence. The 30 $\mu \mathrm{m}$-thick sections were $\mathrm{x} / \mathrm{y}$ scanned along the z-axis with 1-1.5 $\mu \mathrm{m}$ step. Each section was scanned twice: at first with oil immersion $63 \times$ objectives for mapping Herring body distribution and then with an oil immersion $63 \times$ with zoom $2 \times$ objective for evaluating the fine structure of the Herring bodies. Vertical scanning planes were within the whole depth of the section.

The specificity of the antiserum to $\mathrm{TH}$ was determined previously (Arluison et al., 1984). Additional controls for the specificity of antibodies to VP and $\mathrm{TH}$ consisted of either omitting the primary antibodies or preadsorbing them with antigens. The possible cross-reactivity between primary and secondary antibodies was excluded by incubating for each antigen the corresponding mouse primary antibodies with goat anti-rabbit secondary antibodies or, conversely, rabbit primary antibodies with goat anti-mouse secondary antibodies, which resulted in no labeling. The intermediate lobe of the pituitary, being free of VP-immunorective (VP-IR structures, served as an additional physiological control.

In the second series of the immunocytochemical studies, the pituitaries of intact rats, as well as those of rats that were salt-loaded for 1,2 , or 3 weeks (three animals in each group), were cut with a cryostat microtome for serial sections, 14 $\mu \mathrm{m}$ thick. Adjacent sections were mounted on two sets of slides and processed separately for either VP or TH immunocytochemistry. Each slide displayed the sections of an intact animal, as well as those of animals at any period of salt-loading (six sections per animal). The sections were incubated successively with the following: (a) goat normal serum (1\%) for $30 \mathrm{~min}$ at room temperature; (b) rabbit polyclonal antibodies to $\mathrm{TH}$ (diluted 1:2000) or to VP (diluted 1:4000, Chemicon, USA) overnight at $4{ }^{\circ} \mathrm{C}$; (c) biotinylated goat antirabbit antibodies (diluted 1:200, Vector, USA) for $2 \mathrm{~h}$ at room temperature; and (d) an avidin-biotin complex (diluted 1:100, Vector, USA) for $40 \mathrm{~min}$ 
at room temperature. The normal serum and primary and secondary antibodies were diluted in PBS with $0.1 \%$ Triton X-100 (Sigma, USA). The sections were rinsed with PBS after each incubation, except the first. Finally, the sections were rinsed with $0.05 \mathrm{M}$ Tris- $\mathrm{HCl}$ buffer ( $\mathrm{pH} 7.6$ ), and the peroxidase of the avidin-biotin complex was revealed in the same buffer containing $0.05 \%$ 3,3'-diaminobenzidine tetra-hydrochloride (Sigma, USA) and $0.01 \% \mathrm{H}_{2} \mathrm{O}_{2}$. The developing time was constant for each antigen. The sections were dehydrated, mounted in Permount, coverslipped, and then examined with a light microscope.

Carto ${ }^{\circledR}$ software (IMSTAR, France) was used to carry out a quantitative and semi-quantitative examination of VP-IR and TH-IR large axon swellings ( $\geq 6 \mu \mathrm{m}$ in size), Herring bodies, in intact and salt-loaded rats. According to this approach, the relative concentrations of VP and TH could be accurately assessed, and the results obtained at different periods of osmotic stimulation could easily be compared in-between and with those in control animals. Indeed, all preparative procedures of the sections were performed under standardized conditions that allowed us to apply meaningful quantitative and semi-quantitative analysis. Each section in the entire series of the PL was reviewed under a light microscope using a $25 \times$ objective, followed by transferring the image via video camera (CCD) to a computer monitor (IBM PC Pentium 60) and recording.

The relative concentrations of VP-IR and THIR materials were measured as the 'gray level' (GL), related to the optical density (OD) of the specimen, as follows (Conn, 1990):

$\mathrm{OD}_{\text {Specimen }}-\mathrm{OD}_{\text {Background }}=\log \left(\mathrm{GL}_{\text {Background }}\right)-\log \left(\mathrm{GL}_{\text {Specimen }}\right)$

The GL of the background was measured in the vicinity of the PL, namely, in the anterior lobe with no specific immunostaining. The following average parameters of Herring bodies were estimated in intact animals, as well as in each experimental group: (a) OD of VP-IR or TH-IR material in Herring bodies, (b) number of VP-IR or TH-IR Herring bodies per section, (c) area of the individual VP-IR or TH-IR Herring bodies, (d) relative amount of VP-IR or TH-IR material per Herring body $($ area $\times O D)$ as a percentage of its level in the control (intact) animals $(100 \%)$. The profiles of individual Herring bodies were outlined with a light pen to obtain a morphometric mask.

Statistical analysis was carried out using descriptive statistics and the non-parametric Wilcoxon matched pair test.

\section{RESULTS}

\section{Intact Rats}

The PL of intact rats contained an abundant network of VP-IR fibers having transient and terminal swellings. A minor portion of the swellings was represented by Herring bodies, more than $6 \mu \mathrm{m}$ in diameter (Fig. 1a). The Herring bodies were either scattered throughout the PL or concentrated in its proximal portion, as well as along the border with the intermediate lobe. In the individual Herring bodies, VP-IR material was distributed either homogeneously or mainly at the periphery, thereby showing weak immunostaining of the central portion.

The PL of intact animals was also occupied by a network of TH-IR fibers (Fig. 1b), being particularly dense along the intermediate lobe and in the most proximal region of the PL. In contrast to VP-IR fibers, TH-IR fibers possessed swellings that were usually no more than $2 \mu \mathrm{m}$ in size. Nevertheless, occasional, relatively large and intensely immunostained TH-IR swellings were also observed (Figs. 2 to 4). No Herring bodies with colocalization of VP-IR and TH-IR materials were detected in the PL of intact animals when using immunofluorescence double-labeling. 

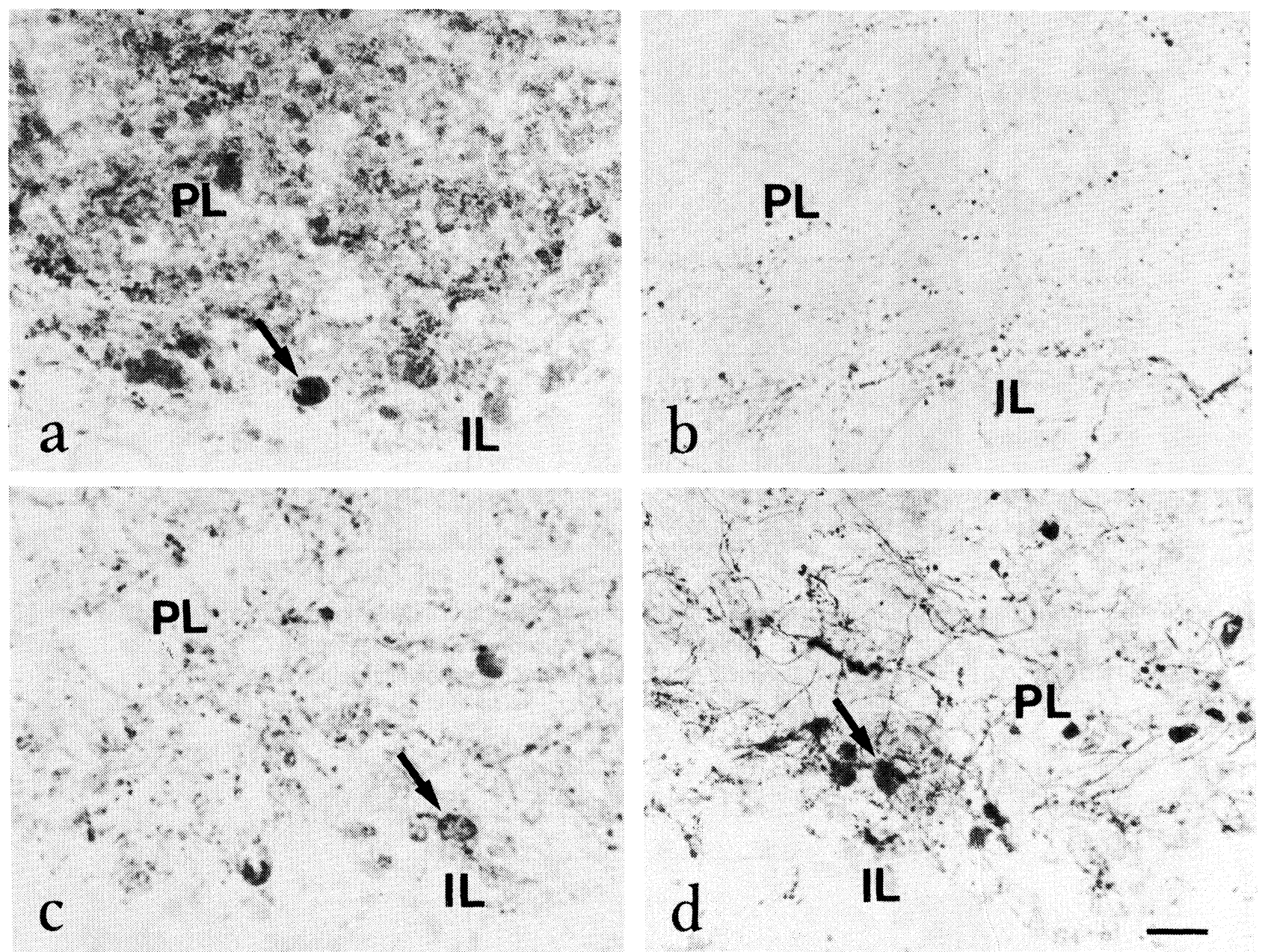

Fig. 1: Vasopressin-immunoreactive $(a, c)$ and tyrosine hydroxylase-immunoreactive (b, d) Herring bodies (arrows) in the posterior lobe (PL) of intact rats ( $a, b)$ and following the osmotic stimulation for a week (c, d). Avidin-biotin technique. IL - intermediate lobe. Scale bar $=20 \mu \mathrm{m}$ for $\mathrm{a}, \mathrm{c}, \mathrm{d} ; 25 \mu \mathrm{m}$ for $\mathrm{b}$.

\section{Rats Salt-Loaded for 1 Week}

Herring bodies, immunoreactive for both VP and $\mathrm{TH}$, were distributed in the same fashion as in the PL of intact rats (Fig. 1c, 1d). They were usually seen in close vicinity of the intermediate lobe and the hypophysial stalk. According to the avidin- biotin immunocytochemical study, the VP-IR Herring bodies decreased dramatically in number (by fourfold) (Fig. 2) and slightly but significantly in size (Fig. 3). Although no change in the OD of VP-IR Herring bodies was observed (Fig. 4), the relative content of VP-IR material per Herring body slightly decreased (Fig. 5). 


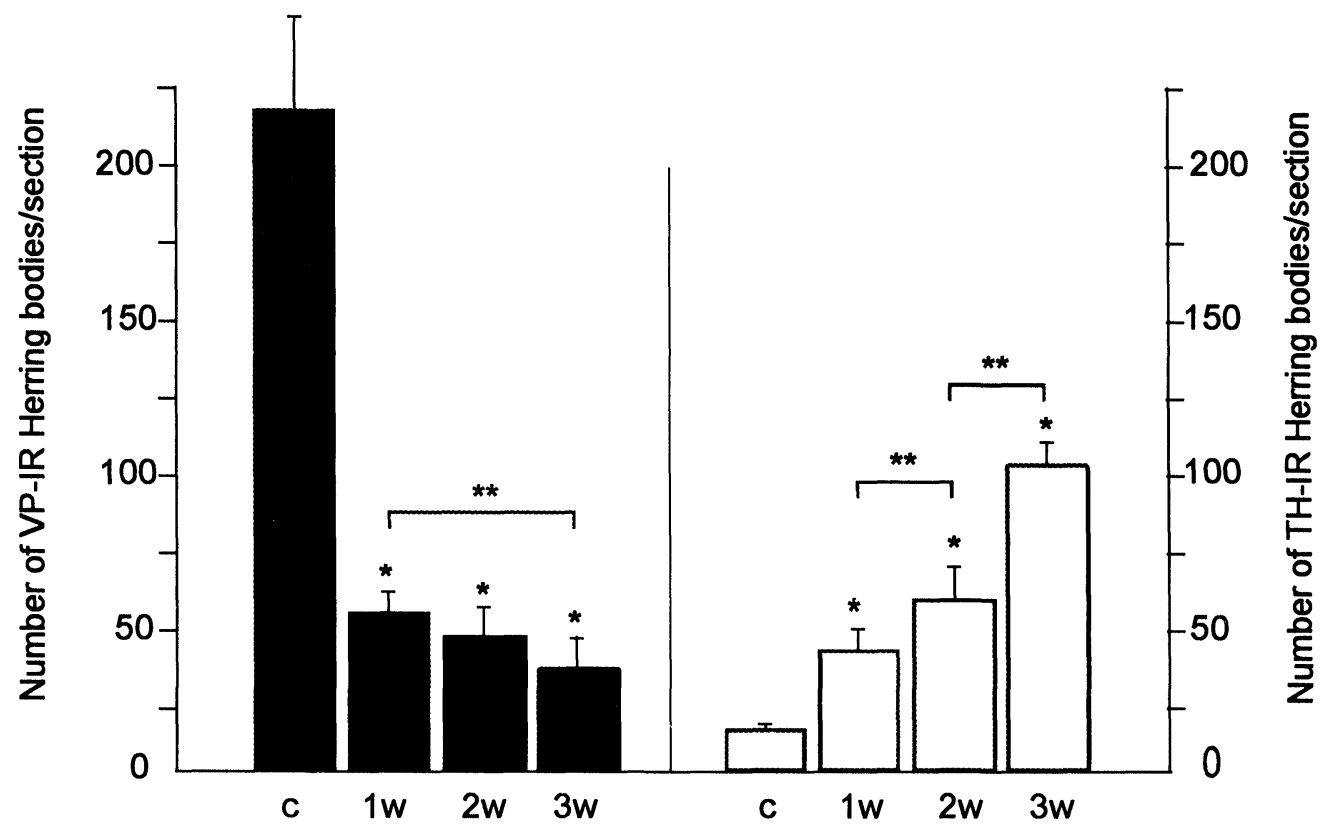

Fig. 2: Number of the vasopressin-immunoreactive (VP-IR) and tyrosine hydroxylase-immunoreactive (TH-IR) Herring bodies in the posterior lobe per section in control (intact) rats (c) and in rats salt-loaded ( $2 \% \mathrm{NaCl})$ for 1,2 and 3 weeks $(\mathrm{w}) .{ }^{*} \mathrm{P}<0.01$, compared to the control. ${ }^{* *} \mathrm{P}<0.01$, comparison between selected groups.

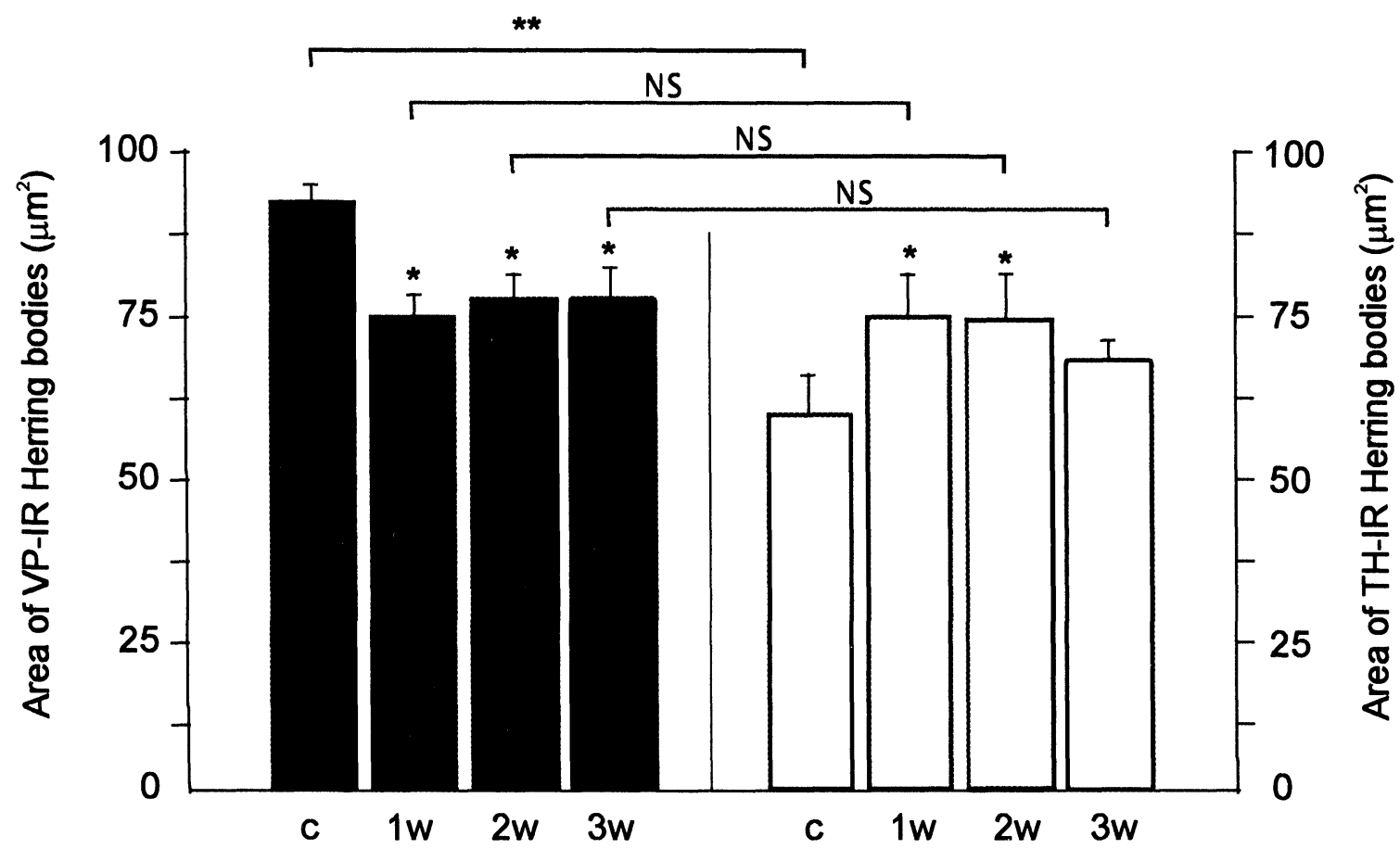

Fig. 3: Area of vasopressin-immunoreactive (VP-IR) and tyrosine hydroxylase-immunoreactive (TH-IR) Herring bodies in the posterior lobe in control (intact) rats $(\mathrm{c})$ and in rats salt-loaded $(2 \% \mathrm{NaCl})$ for 1,2 and 3 weeks $(\mathrm{w})$. $^{*} \mathrm{P}<0.01$, compared with the control. $* * \mathrm{P}<0.01$, comparison between selected groups. NS, non significant. 


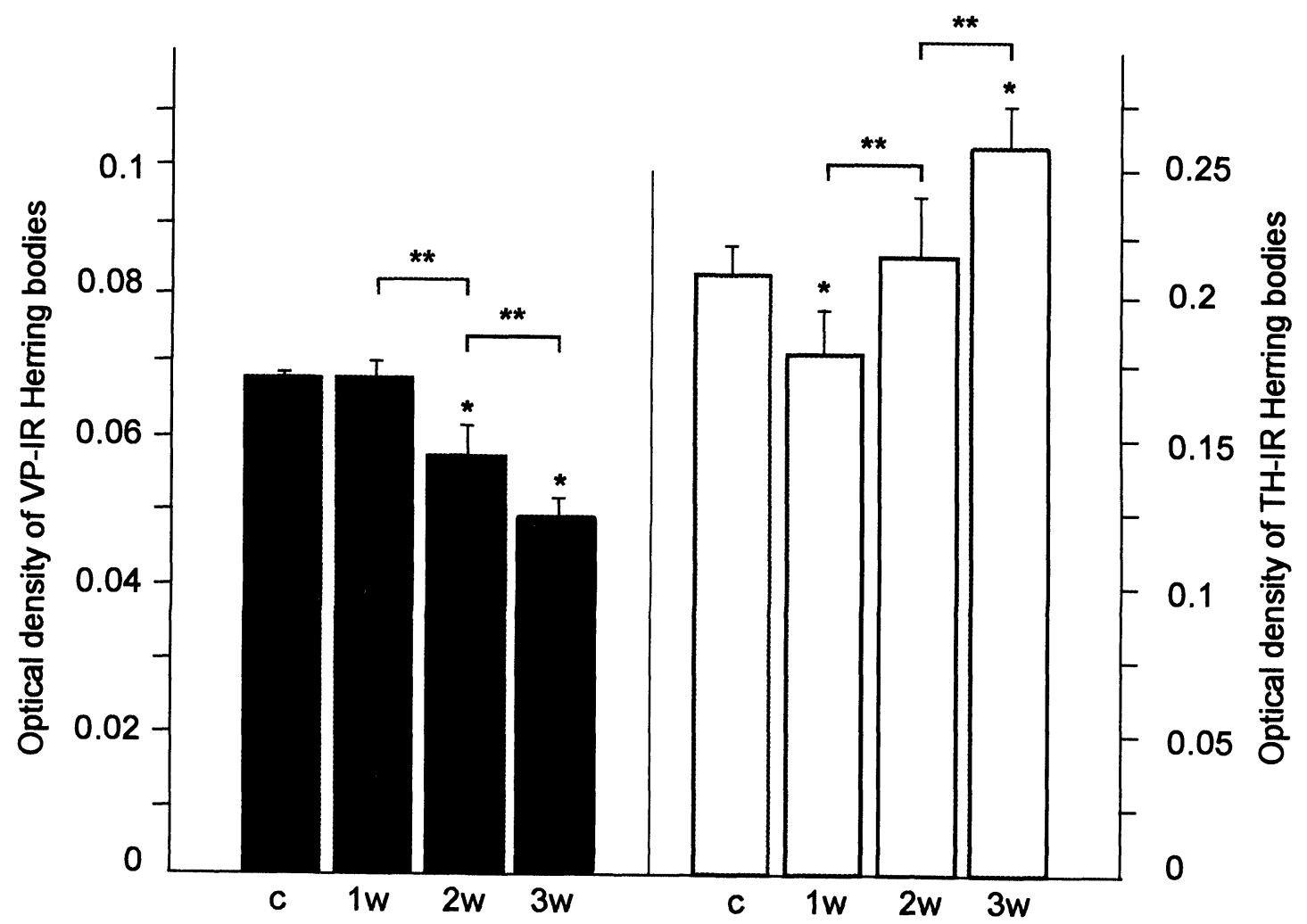

Fig. 4: Optical density of vasopressin-immunoreactive (VP-IR) and tyrosine hydroxylase-immunoreactive (TH-IR) Herring bodies in the posterior lobe in control (intact) rats $(\mathrm{c})$ and in rats salt-loaded $(2 \% \mathrm{NaCl})$ for 1,2 and 3 weeks $(\mathrm{w}) .{ }^{*} \mathrm{P}$ $<0.01$, compared to the control. ${ }^{* *} \mathrm{P}<0.01$, comparison between selected groups.

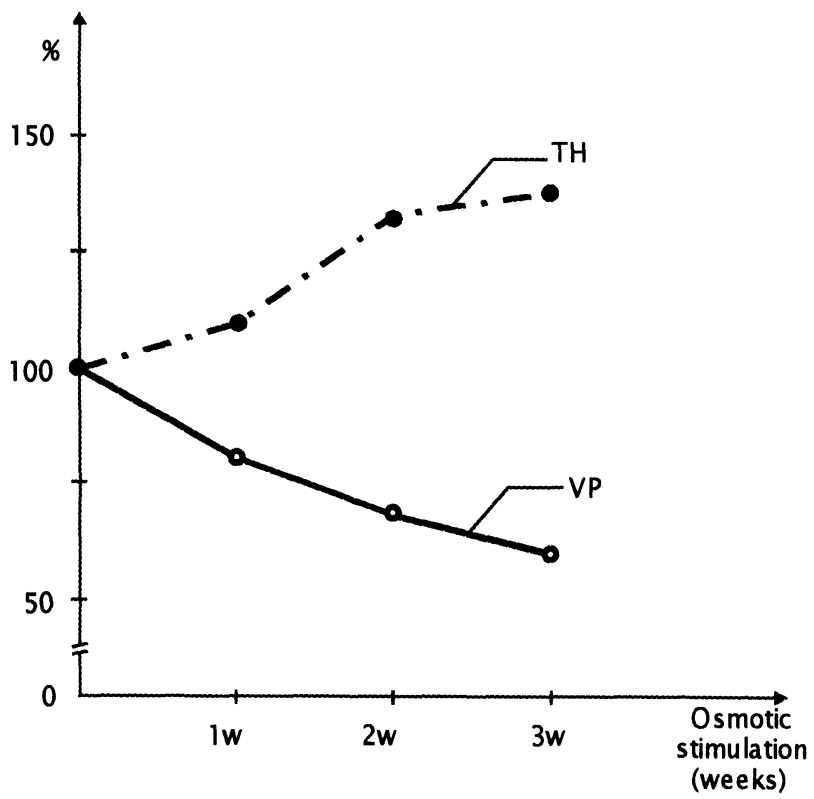

Fig. 5: Relative amount of vasopresin (VP) and tyrosine hydroxylase (TH) per Herring body in control rats and rats saltloaded for 1, 2 and 3 weeks (w). The results are presented as percentage of the control level (100\%). 

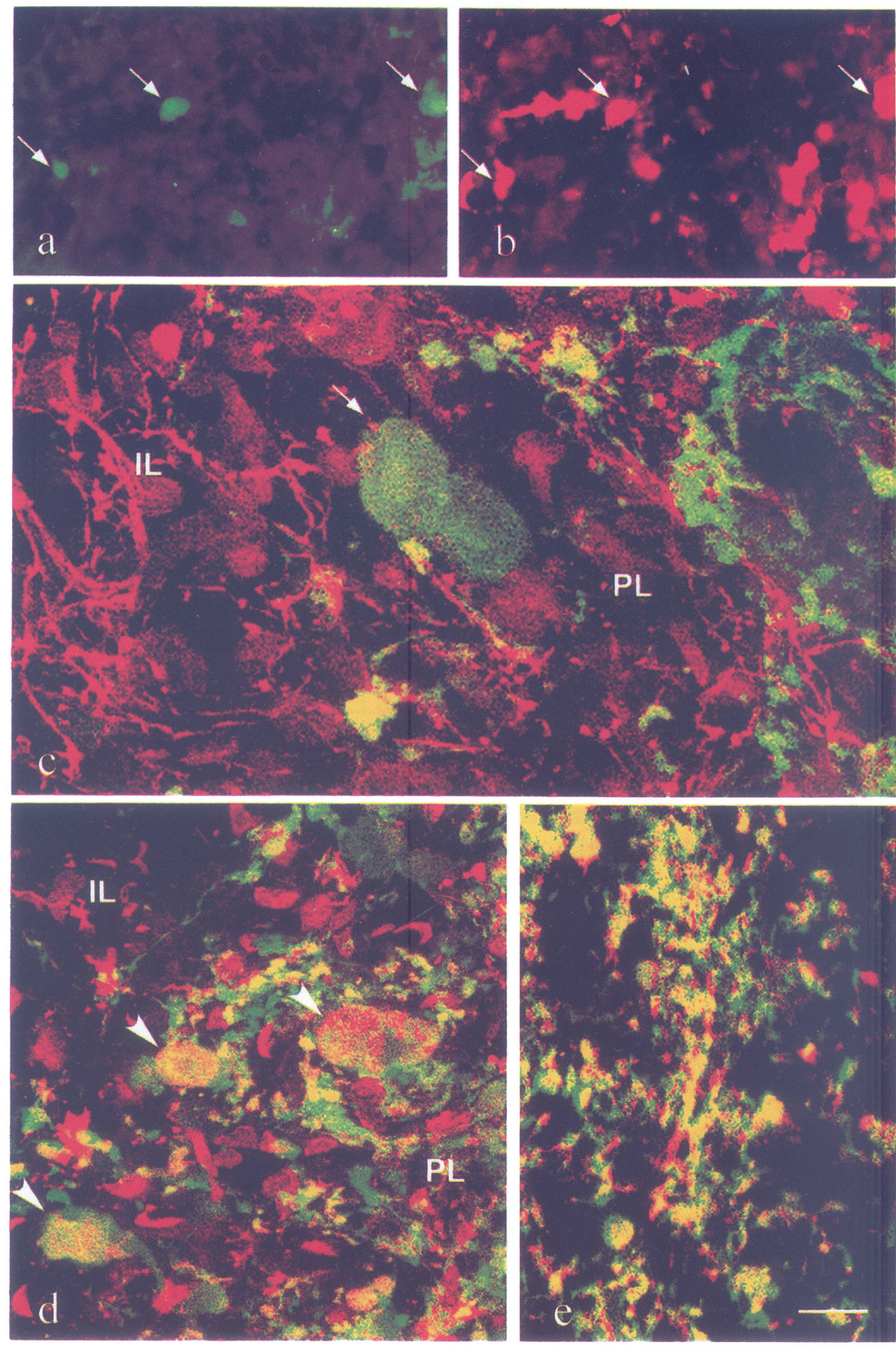

Fig. 6: Herring bodies (a, b, c, d) and small axon swellings (e) immunoreactive for vasopressin (rhodamine, red for b; fluorescein, green for c, d, e), tyrosine hydroxylase (fluorescein, green for a; rhodamine, red for c, d, e) as well as for both vasopressin and tyrosine hydroxylase (yellow for $\mathrm{c}, \mathrm{d}, \mathrm{e}$ ) in the posterior lobe (PL) of rats salt-loaded for a week. a, b, fluorescent microscopy; c-e, confocal microscopy. IL, intermediate lobe. Arrows, vasopressin- and/or tyrosine hydroxylase-immunoreactive Herring bodies; arrowheads, double-labeled Herring bodies with zonality in the distribution of the immunoreactive material. Scale bar $=20 \mu \mathrm{m}$ for $\mathrm{a}, \mathrm{b} ; 6 \mu \mathrm{m}$ for $\mathrm{c}$ and $8 \mu \mathrm{m}$ for $\mathrm{d}$, e. 
In contrast to the VP-IR Herring bodies, the number (Fig. 2) and the average area (Fig. 3) of the TH-IR Herring bodies increased considerably, which was accompanied by an increase of the amount of the TH-IR material per Herring body (Fig. 5).

The double-immunofluorescence study showed that numerous VP-IR Herring bodies contained TH-IR material (Fig. 6a, b), although monolabeled VP-IR (Fig. 6c), and TH-IR were also observed. The distribution of VP-IR and TH-IR materials in some Herring bodies was not homogeneous (Fig. 6d). The VP-IR material was often distributed at the periphery of Herring bodies. In addition to Herring bodies, double-immunolabeled thin fibers with small swellings $(<1.5 \mu \mathrm{m})$ were regularly seen in a confocal microscope (Fig. 6e).

\section{Rats Salt-Loaded for Two Weeks}

Over the second week of osmotic stimulation, no change in the number and size of the VP-IR Herring bodies was observed (Figs. 2, 3), whereas their OD and the content of the VP-IR material per Herring body slightly decreased (Figs. 4, 5). In contrast to the VP-IR Herring bodies, the number (Fig. 2) and OD (Fig. 4) of TH-IR Herring bodies, as well as the content of the TH-IR material per Herring body (Fig. 5), increased significantly. The average area of the TH-IR Herring bodies did not change during the second week of osmotic stimulation (Fig. 3).

\section{Rats Salt-Loaded for Three Weeks}

During the third week of osmotic stimulation, the number, size, and OD of the VP-IR Herring bodies did not change (Figs. 2 to 4 ), whereas the content of the VP-IR material per Herring body slightly decreased. The same was true for the average area of the TH-IR Herring bodies (Fig. 3), whereas their numbers (Fig. 2), OD (Fig. 4), and content of TH-IR material in the individual Herring bodies increased considerably.

\section{DISCUSSION}

\section{Technical Remarks}

In this study, a double-immunofluorescence technique was applied to determine whether $\mathrm{TH}$ is an attribute not only of the perikarya but also of the distal axons of stimulated magnocellular VPergic neurons. Moreover, an image analysis made it possible to specify VP-IR and TH-IR Herring bodies in rats, under normal laboratory conditions and following 1, 2, or 3 weeks of saltloading, by measuring the number of Herring bodies, their individual area, and the OD of IR material. We have emphasized (see Experimental Methods) that the PL of intact rats and of those at any stage of salt-loading were processed under standardized conditions (fixation, cutting, immunostaining of the sections mounted on the same slides) for quantitative studies and particularly for the semi-quantitative analysis of TH-IR and VP-IR materials. The validity of this approach was proved in earlier technical studies (Conn, 1990) and then confirmed by concrete applications (Beltramo et al., 1994, 1997). Still, it should be taken into account that we could compare only the relative intracellular amounts of the IR materials in the animals of different experimental groups, but separately for TH-IR and VP-IR materials. In other words, this technique failed to evaluate the absolute content of the IR material and to compare the OD or the relative amounts of TH-IR and VPIR materials in Herring bodies.

This is the first study attempting to compare the dynamics of the TH and VP turnover in the axons of VPergic neurons of the supraoptic 
nucleus during long-term osmotic stimulation by using avidin-biotin immunocytochemistry and image analysis. When evaluating the intra-axonal dynamics of TH and VP, it should be taken into account that these substances differ considerably in some specific properties. Thus, TH is a nonreleasable cytosolic protein that is degraded by cytosolic peptidases (Fernandez \& Craviso, 1999). Conversely, VP is a secreted peptide that is stored in secretory granules and protected from enzymatic degradation by the granule membrane (Castel et al., 1984). From the above comments follows that the relative average concentration (OD) or the content (ODxarea of a Herring body) of TH-IR material per Herring body is proportional to the difference between the TH transport from the cell bodies of VPergic neurons and the enzymatic degradation of TH in Herring bodies. In turn, the relative average concentration (OD) or content (ODxarea of a Herring body) of the VP-IR material per Herring body is proportional to the difference between VP transport from the cell bodies of VPergic neurons and VP depletion from these structures. The relative contents of polypeptides (IR materials) per Herring body appeared to be a more informative index for their turnover than their relative concentrations were because the Herring body size changed during the course of the experiment. For instance, the relative concentration of the VP-IR material (OD) in Herring bodies did not change for the first week of osmotic stimulation, whereas the relative content of the VP-IR material per Herring body decreased over the same period (see Results).

\section{Colocalization of TH and VP in Herring Bodies}

In this study, we have demonstrated for the first time a colocalization of TH and VP in Herring bodies in the PL by using an immunofluorescence double-labeling technique. In addition to Herring bodies, small double-labeled axon swellings were seen regularly in the PL of the rats that were saltloaded for a week when using confocal microsocopy. The data strongly suggest that $\mathrm{TH}$ is transported from the cell bodies located in the supraoptic nucleus toward the axon terminals in the PL. Although TH mRNA has been detected before in the VPergic axons in the median eminence on sections (Skutella et al., 1994) and in the homogenate of the 'neurointermediate' (PL + intermediate lobe) lobe (Melia et al., 1994), the authors are skeptical about the possible translation of TH mRNA in the axon terminals because of the absence of translational machinery in this neuronal compartment.

Although both intact rats and those that were salt-loaded for a week were tested for colocalization of TH and VP, double-labeled Herring bodies were detected only in the salt-loaded animals. Apparently, this result is accounted for by the extremely low frequency of TH-containing Herring bodies in intact rats, whereas their number significantly increased for the first week of osmotic stimulation (see Results). The same was also true for VPergic cell bodies expressing TH gene and synthesis in rats during the first week of salt-loading (Abramova et al., unpublished).

Surprisingly, some double-labeled Herring bodies did not show full superposition of the TH-IR and VP-IR profiles. Moreover, VP-immuno-staining of some Herring bodies was stronger at the periphery than in the center. These findings appear to agree with earlier electron microscopic observations of the zonality in the distribution of subcellular structures in Herring bodies-the VP-containing secretory granules occupied the periphery, whereas the central area was filled with microtubules (Dellmann \& Rodriguez, 1970; Polenov \& Garlov, 1971; Ugrumov \& Belenkii, 1974).

It is noteworthy that VP-IR Herring bodies decreased in size for the first week of osmotic stimulation, while TH-IR Herring bodies increased 
in size during the same period, becoming as large as VP-IR Herring bodies. After that, the Herring bodies immunostained for TH or VP did not change in size until the end of the experiment. These observations might be a result of two synchronous processes: (1) a sharp depletion of the peripheral area of Herring bodies from the VP-containing secretory granules that perhaps was followed by the shrinkage of Herring bodies, and (2) a spread of the TH-IR material from the center to the periphery of Herring bodies, due to its diffusion either through axoplasm or via the hypertrophied microtubular system. Indeed, the concentration of intra-axonal microtubules increases under functional stimulation of the VP magnocellular system (Knowles \& Vollrath, 1974).

\section{TH and VP Turnover in Herring Bodies}

In the present study, peculiar attention was paid to VP and TH turnover in Herring bodies during the long-term osmotic stimulation of VPergic neurons. The number of VP-IR Herring bodies decreased fourfold over the first week of osmotic stimulation, remaining almost at the same low level until the end of the experiment. In other words, the depletion of VP from Herring bodies appeared to occur at a higher rate when compared with the depletion of the VP axons in the PL as a whole (Abramova et al., unpublished), which contradicts earlier qualitative studies (Ugrumov \& Belenkii, 1974). The relative content of VP-IR material within individual Herring bodies fell gradually over the entire period of osmotic stimulation studied here, whereas its concentration decreased only for the second and the third week. This result suggests that the VP depletion from Herring bodies prevailed during its transport from the cell bodies for the whole experiment. In contrast to VP-IR Herring bodies, the number of TH-IR Herring bodies progressively increased during the 3 weeks of salt-loading. This phenomenon is apparently a consequence of the gradually increasing number of VPergic neurons co-expressing TH (Abramova et al., unpublished) and finally, of the transport of TH in these neurons from the perikarya toward axon terminals.

The functional significance of TH in magnocellular VPergic neurons remains unclear. The VPergic cell bodies in the supraoptic nucleus lacked the specific markers of TH enzymatic activity: guanosine triphosphate-cyclohydrolase, the enzyme cathalizing the TH co-factor production $\left(\mathrm{BH}_{4}\right)$, and L-DOPA, the immediate precursor of dopamine (Marsais \& Calas, 1999). Moreover, one cannot exclude the superfluous gene expression of $\mathrm{TH}$ as that of tryptophan hydroxylase in the neurons of the zona incerta (Vanhatalo \& Soinila, 1996). Still, the synchronization of gradual VP depletion and $\mathrm{TH}$ accumulation in Herring bodies during the entire period of long-term osmotic stimulation studied here raises the question of the functional interaction between VP and TH in axon terminals.

Thus, the VPergic axons of osmotically stimulated neurons contained TH. The VP release from Herring bodies gradually increased during longterm osmotic stimulation and was synchronized with $\mathrm{TH}$ accumulation, at least for the second and third weeks. This correlation suggests functional interactions between VP and TH in axon terminals.

\section{ACKNOWLEGMENTS}

The present study was supported by the following grants: PICS (98-04-22018), NATO (OUTR.CRG 970131), INTAS-RFBR (95-IN-RU1246), RFBR (96-04-49441), French Ministry of Education and Science and Ministry of Foreign Affairs. The authors express their thanks to Dr. Ph. Mailly (Groupe Reseau et Imagerie, UFR des Sciences de la Vie, Université P. et M. Curie, Paris, France) for the expert assistance in the image analysis. 


\section{REFERENCES}

Arluison M, Dietl M, Thibault J. Ultrastructural morphology of dopaminergic nerve terminals and synapses in the striatum of the rat using tyrosine hydroxylase immunocytochemistry: A topographical study. Brain Res Bull 1984; 13: 169-185.

Beltramo M, Calas A, Chernigovskaya E, Borisova N, Polenova $\mathrm{O}$, Tillet $\mathrm{Y}$, et al. Postnatal development of the suprachiasmatic nucleus in the rat. Morphofunctional characteristics and time course of tyrosine hydroxylase immunopositive fibers. Neuroscience 1994; 63: 603-610.

Beltramo M, Calas A, Chernigovskaya E, Thibault J, Ugrumov M. Long-lasting effect of catecholamine deficiency on differentiating vasopressin and oxytocin neurons in the rat supraoptic nucleus. Neuroscience 1997; 79: 555-561.

Brownstein MJ, Mezey E. Multiple chemical messengers in hypothalamic magnocellular neurons. Prog Brain Res 1986; 68: 161-168.

Castel M, Gainer H, Dellmann HD. Neuronal secretory systems. Int Rew Cytol 1984; 88: 303-458.

Conn PM, ed. Methods in Neurosciences. Quantitative and Qualitative Microscopy; Image Analytic Techniques for Quantification of ImmunohistoChemical Staining in the Nervous System. San Diego, California, USA: Academic Press, 1990.

Dellmann HD, Rodriguez EM. Herring bodies: an electron microscopic study of local degeneration and regeneration of neurosecretory axons. Z Zellforsch 1970; 111:293-315.

Fernandez E, Craviso GL. Protein synthesis blockade differentially affects the degradation of constitutive and nicotinic receptor-induced tyrosine hydroxylase protein level in isolated bovine chromaffin cells. J Neurochem 1999; 73: 169-178.

Fetissov S, Marsais F, Nicolaïdis S, Calas A. Expression of tyrosine hydroxylase in magnocellular hypothalamic neurons of obese (fa/fa) and lean heterozygous $(\mathrm{Fa} / \mathrm{fa})$ Zucker rats. Mol Brain Res 1997; 50: 314-318.

Hökfelt T, Meister B, Melander T, Everitt B. Coexistence of Classical Transmitters and Peptides with Special Reference to the Arcuate NucleusMedian Eminence Complex. Hypothalamic Dysfunction in Neuropsychiatric Disorders. New York, NY, USA: Raven Press, 1987.

Kawano H, Daikoku S. Functional topography of the rat hypothalamic dopamine neuron systems: retrograde tracing and immunohistochemical study. J Comp Neurol 1987; 265: 242-253.

Kiss JZ, Mezey E. Tyrosine hydroxylase in magnocellular neurosecretory neurons. Neuroendocrinology 1986; 43: 519-525.

Knowles F, Vollrath L, ed. Neurosecretion - The Final Neuroendocrine Pathway; Overactivity of the Hypothalamo-Neurohypophysial Neurosecretory System and the Problem of Mechanisms of Transporting Neurosecretory Material. Berlin, Germany: Springer, 1974.

Marsais F, Calas A. Ectopic expression of noncatecholaminergic tyrosine hydroxylase in rat hypothalamic magnocellular neurons. Neuroscience 1999; 94: 151-161.

Meister B, Villar MJ, Ceccatelli S, Hökfelt T. Localization of chemical messengers in magnocellular neurons of the hypothalamic supraoptic and paraventricular nuclei: An immunohistochemical study using experimental manipulations. Neuroscience 1990a; 37: 603-633.

Meister B, Cortes R, Villar MJ, Schaulling M, Hökfelt $T$. Peptides and transmitter enzymes in hypothalamic magnocellular neurons after administration of hyperosmotic stimuli: Comparison between messenger RNA and peptide-protein levels. Cell Tissue Res 1990b; 260: 279-298.

Melia KR, Trembleau A, Oddi R, Sanna PP, Bloom FE. Detection and regulation of tyrosine hydroxylase nRNA in catecholaminergic terminals fields: Possible axonal compartmentalization. Exp Neurol 1994; 130: 394-406.

Nagatsu I, Sakai M, Takeuchi T, Arai R, Karasawa N, Yamada K, Nagatsu T. Tyrosine hydroxylase (TH)only-immunoreactive non-catecholaminergic neurons in the brain of wild mice or the human $\mathrm{TH}$ transgenic mice do not contain GTP cyclohydrolase I. Neurosci Lett 1997; 228: 55-57.

Polenov AL, Garlov PE. The hypothalamo-hypophyseal system in Acipenseridae. I. Ultrastructural organization of large neurosecretory terminals (Herring bodies) and axoventricular contacts. Z Zellforsch 1971; 116: 349-374.

Robert FR, Leon-Henri BP, Chapleur-Chateau MM, Girr MN, Burlet AJ. Comparison of three immunoassays in the screening and characterization of monoclonal antibodies against arginine-vasopressin. J Neuroimmunol 1985; 9 (3-4): 205-20.

Saavedra JM, Palkovits M, Kizer JS, Brownstein M, Zivin JA. Distribution of biogenic amines and 
related enzymes in the rat pituitary gland. $J$ Neurochem 1975; 25: 257-260.

Saavedra JM. Central and peripheral catecholamine innervation of the rat intermediate and posterior pituitary lobes. Neuroendocrinology 1985; 40: 281-284.

Skutella T, Probst JS, Blanco E, Jirikowski GF. Localization of tyrosine hydroxylase mRNA in the axons of the hypothalamo-neurohypophysial system. Mol Brain Res 1994; 23; 179-184.

Seil FJ, Johnson ML, Nishi R, Nilaver G. Tyrosine hydroxylase expression in non-catecholaminergic cells in cerebellar cultures. Brain Res 1992; 569: 164-168.

Ugrumov MV, Belenkii MA. Electron microscope investigation of the large swelling of neurosecretory fibres (the Herring bodies) in the posterior pituitary of chronically dehydrated albino rats. Tsytologia
1974; 16: 281-286 (in Russian).

Vanhatalo S, Soinila S, Kaartinen K, Bäck N. Colocalization of dopamine and serotonin in the rat pituitary gland and in the nuclei innervating it. Brain Res 1995; 669: 275-284.

Vanhatalo S, Soinila S. Superfluous expression of tryptophan hydroxylase in the zona incerta dopaminergic neurones. Neuroreport 1996; 7: 28892892.

Verney C, Gaspar P, Febvret A, Berger B. Transient tyrosine hydroxylase-like immunoreactive neurons contain somatostatin and substance $P$ in the developing amygdala and bed nucleus of the stria terminalis of the rat. Brain Res 1988; 470: 45-58.

Young WS, Warden M, Mezey E. Tyrosine hydroxylase mRNA is increased by hyperosmotic stimuli in the paraventricular and supraoptic nuclei. Neuroendocrinology $1987 ; 46: 439-444$. 

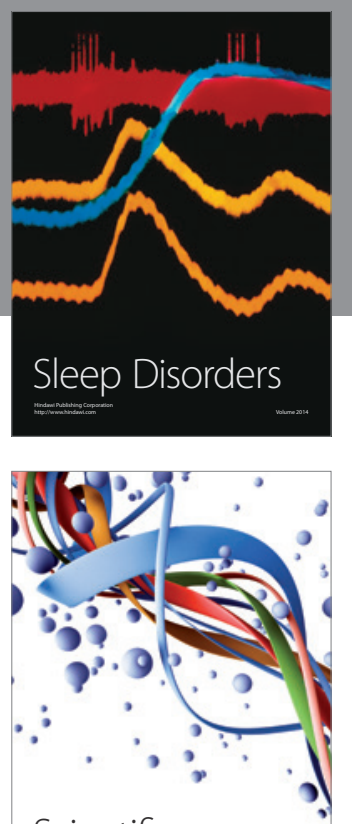

Scientifica
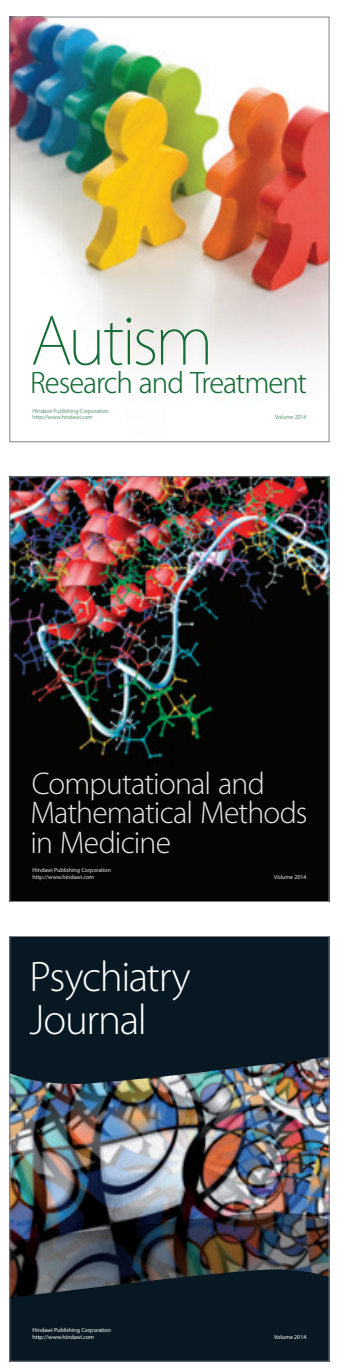
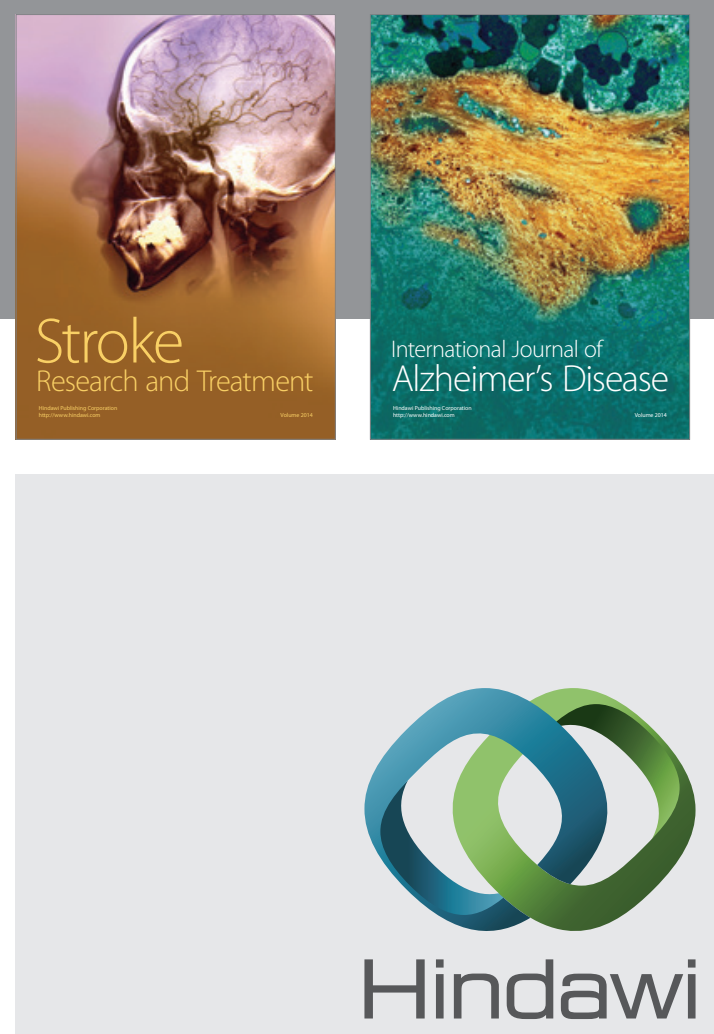

Submit your manuscripts at

http://www.hindawi.com
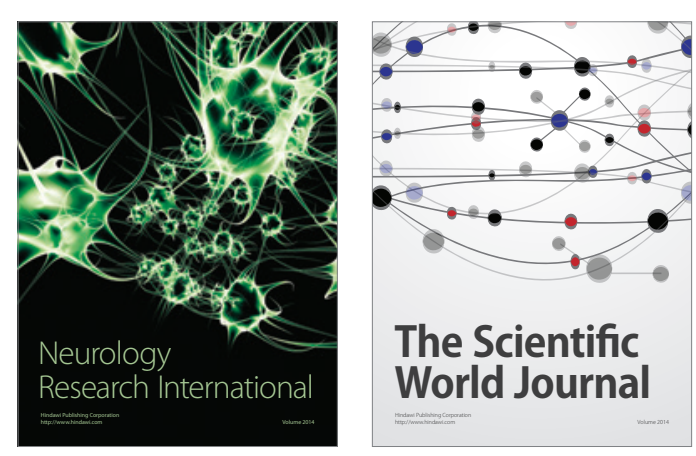

The Scientific World Journal

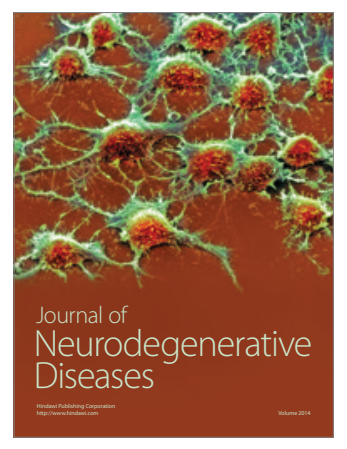

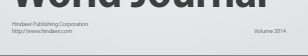

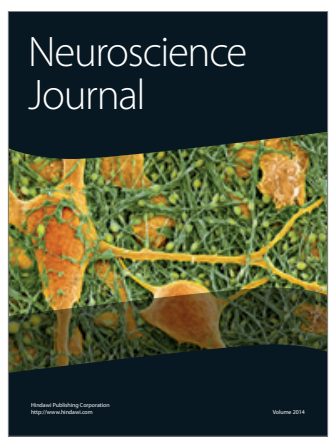

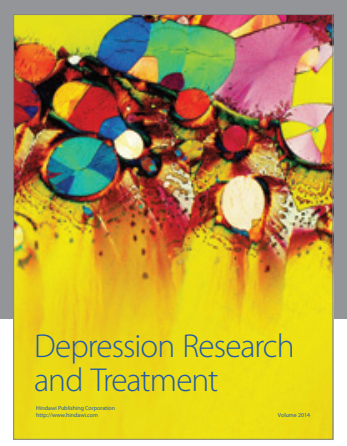
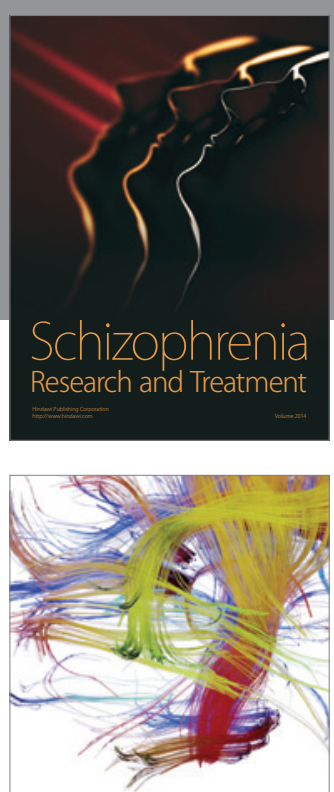

Brain Science

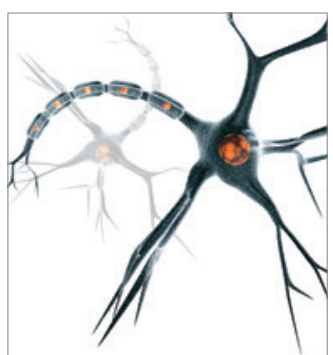

Neural Plasticity
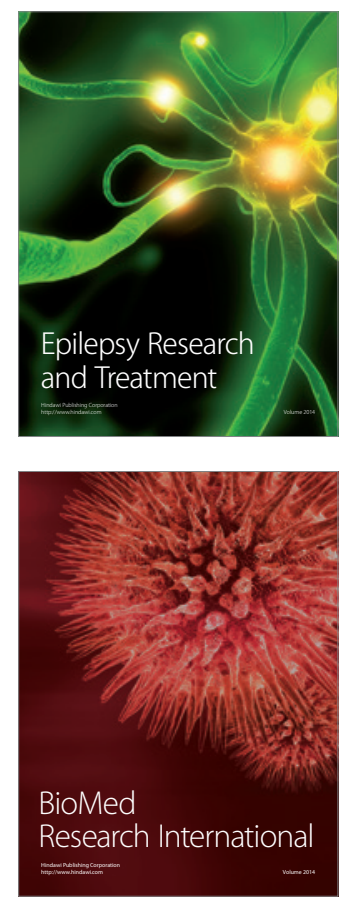

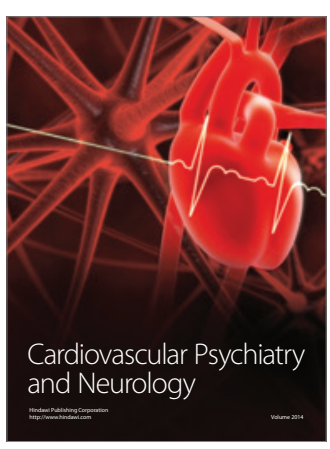

Parkinson's

Disease
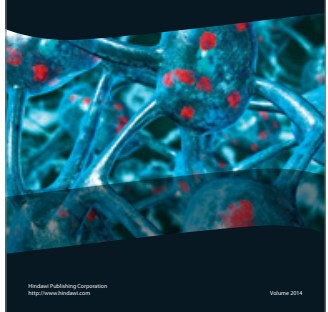